\title{
CARTESIAN CONTROL OF REDUNDANT ROBOTS
}

\author{
R. Colbaugh \\ K. Glass \\ New Mexico State University, Las Cruces, NM. 88003
}

\begin{abstract}
This paper presents a Cartesian-space position/force controller for redundant robots. The proposed control structure partitions the contrul problem into a nonredundant position/force trajectory tracking problem and a redundant mapping partion problem between Cartesian control input $F$ map is exploited so that the robot redundancy is utilized to improve the dynamic response of the robot. This dynamically optimal $F \rightarrow T$ map is implemented locally (in time) so that it is computationally reficient for on-line control; however, it is shown that the map possesses globally optimal characteristics. Additionally, it is efficient for on-line control; however, it is $F \rightarrow T$ map can be modified so that the robot redundancy is used to simultaneously

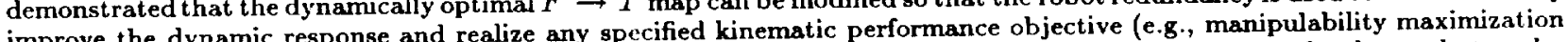
or obstacle avoidance). Computer simulation results are given for a four degree of freedom planar redundant robot under Cartesian control, and demonstrate that position/force trajectory tracking and effective redundancy utilization can be achieved simultaneously with the proposed controller.
\end{abstract}

\section{INTRODUCTION}

It is predicted that in the near future robot manipulators will be required to perform complex tasks that demand great dexterity and versatility in both position control and force control applications. Such tasks will require performance superior to that obtainable with conventional six degree of freedom (DOF) robots under the control of joint-space position servo loops. This fact has motivated increased research activity in the area of redundant robot manipulators. Redundant robots possesses This fact has motivated increased achieve the desired position and orientation of the end-effector, and it is expected that the more DOF than are necessary to achieve the desired position and orientation

Most of the research on the control of redundant robots reported to date has focused on the inverse kinematics problem, which involves the calculation of the joint-space trajectory that provides the desired end-effector motion and in addition satisfies some side criterion. The majority of this work has involved using redundancy to realize some kinematic performance objective. A partial list of kinematic performance criteria that have been studied includes singularity avoidance [1,2], obstacle aveidance $[3,4]$, joint limit avoidance $[5,6]$, repetitive motion conservation [6,7], and achievable accuracy [8]. Research in which avoidance $[3,4]$, joint limit avoidance $[5,6]$, repetitive motion conservance objective has been more limited, and includes studies manipulator redundancy is utilized to acheve a dinimizing manipulator energy consumption $[11,12]$, and increasing the robot's dynamic response $[13,14]$.

It has only comple redundant robot control problem [14-19]. The controllers described in [14-16] are model-based control schemes which require complete knowledge and calculation of the complex robot dynamic model. In addition, each of these control algorithms requires either explicit or implicit calculation of the inverse kinematics of the robot. Alternatively, the control strategy presented in [17-19] is an adaptive Cartesian-space control algorithm which does not require calculation of either the robot dynamic model or the inverse kinematics, and which has been apelied only to control problems in which the redundancy is utilized to realize kinematic performance objectives.

This paper presents an adaptive Cartesian position/force controller for redundant robots. The proposed control strategy is to partition the control problem into a nonredundant trajectory tracking problem and a redundant mapping problem between Cartesian control input $F \in R^{m}$ and robot actuator torque $T \in R^{n}$ (for redundant robots, $m<n$ ). The underdetermined nature of the $F \rightarrow T$ map is exploited to allow the redundancy to be effectively utilized directly in Cartesian-space. Computer simulation results are given for a four DOF planar redundant robot under the control of the proposed algorithm, and demonstrate that accurate position/force trajectory tracking and effective utilization of redundancy can be achieved simultaneously with the controller.

The paper is organized as follows. In Section 2, the redundant robot position/force control problem is formulated in the partitioned form indicated above. The $F \rightarrow T$ map which uses the robot redundancy to increase the robot's dynamic response is constructed in Section 3. This dynamically optimal $F \rightarrow T$ map is modified in Section 4 so that the robot redundancy can be used to simultaneously improve the dynamic response and realize any specified kinematic performance objective. The performance of the controller is illustrated in Section 5 through a computer simulation study. Section 6 summarizes the paper and draws some conclusions.

\section{PROBLEM FORMULATION}

\subsection{Basic Theory}

Consider an $n$ DOF robot manipulator performing tasks in an $m$-dimensional Cartesian-space (with $m<n$ ). These tasks will, in general, involve motion of the robot end-effector in certain directions and exertion of force by the end-effector on the environment in the remaining directions. The particular directions of motion and force exertion depend on the nature of the task Consider now a task-related "constraint frame" which is defined by the particular end-effector/environment contact situation [20]. In the constraint frame, the $m$-dimensional Cartesian-space can be decomposed into an $l$-dimensional "position 
subspace" and a $j$-dimensional "force subspace", where $l+j=m$ and where the position subspace and force subspace are orthogonal. The position subspace contains the $l$ directions in which the robot end-effector is free to move and along which the end-effector position is to be controlled, while the force subspace contains the $j$ directions in which the robot end-effector is constrained by the environment and along which the contact force is to be controlled. For convenience it will be assumed in the following that all quantities are expressed in terms of the constraint frame unless otherwise noted.

Let $y \in R^{m}$ define the position (and orientation) of the end-effector in Cartesian-space and $\theta \in R^{n}$ be the vector of joint coordinates. The relationship between end-effector position $y$ and joint-space position $\theta$ is

$$
\begin{aligned}
& y=f(\theta) \\
& \dot{y}=J(\theta) \dot{\theta}
\end{aligned}
$$

where $f: R^{n} \rightarrow R^{m}$ represents the forward kinematics of the robot and $J=\partial f / \partial \theta \in R^{m \times n}$ is the end-effector Jacobian matrix. It may be assumed without loss of generality that the elements of $y$ are ordered such that $y=\left[x^{T} \mid z^{T}\right]^{T}$, where $x \in R^{l}$ and $z \in R^{j}$ are the end-effector position (and orientation) vectors in the position subspace and force subspace, respectively. Given this partitioning for $y$, the following partitioning for $J$ may be defined:

$$
\left[\begin{array}{c}
\dot{x} \\
-- \\
\dot{z}
\end{array}\right]=\left[\begin{array}{c}
J_{p}(\theta) \\
-\bar{\theta}(\theta)
\end{array}\right] \dot{\theta}
$$

where $J_{p} \in R^{1 \times n}$ and $J_{f} \in R^{j \times n}$ are termed the "position subspace Jacobian" and "force subspace Jacobian", respectively. The dynamic model of the robot with its end-effector in contact with the environment may be written in joint-space as [e.g.,
21]

$$
T=H(\theta) \ddot{\theta}+V_{c c}(\theta, \dot{\theta})+V_{f}(\theta, \dot{\theta})+G(\theta)+J_{f}^{T}(\theta) P
$$

where $T \in R^{n}$ is the vector of actuator torques and/or forces, $H \in R^{n \times n}$ is the robot inertia matrix, $P \in R^{j}$ is the end-effector/environment contact force and/or moment, and $V_{c c}, V_{f}, G \in R^{n}$ represent the torque vectors due to Coriolis and centripedal acceleration, friction, and gravity, respectively. Alternatively, the robot dynamic model can be expressed in
Cartesian-space as [e.g., 16]

$$
F=\left(J H^{-1} J^{T}\right)^{-1}[\ddot{y}-\dot{J} \dot{\theta}]+\left(J H^{-1} J^{T}\right)^{-1} J H^{-1}\left[V_{c c}+V_{f}+G\right]+P^{*}
$$

where $F \in R^{m}$ is the generalized force vector corresponding to the generalized coordinate $y$, and $P^{*}=\left[0^{T} \mid P^{T}\right]^{T} \in R^{m}$ with the zero denoting an $l$-dimensional zero vector.

The general Cartesian-space position/force control problem for the redundant robot described in (1)-(3) may be considered to consist of two steps:

1.) Cartesian position/force trajectory tracking:

compute the Cartesian control input $F=\left[F_{p}^{T} \mid F_{f}^{T}\right]^{T} \in R^{m}$ required to track the desired $m$-dimensional position/force trajectory, where $F_{p} \in R^{l}$ is the position control input that tracks the desired end-effector position trajectory $x_{d} \in R^{l}$ and $F_{j} \in R^{j}$ is the force control input that tracks the desired end-effector/environment contact force trajectory $P_{d} \in R^{j}$

2.) $F \rightarrow T$ mapping:

compute the joint torque vector $T \in R^{n}$ required to realize $F$ while simultaneously accomplishing some desired kinematic and/or dynamic performance objective.

Each of the steps will now be considered individually.

\subsection{Cartesian Position/Force Trajectory Tracking}

Observe that the Cartesian position/force trajectory tracking problem is nonredundant since $F_{p}$ and $x_{d}$ are both of dimension $l$ and $F F_{f}$ and $P_{d}$ are both of dimension $j$. Thus many different control strategies could be improvised to compute the control input $F$ that would ensure that the dynamics (3b) tracks the desired end-effector position/force trajectory. Here the adaptive Cartesian-space position/force controller recently developed by Seraji [22] for nonredundant robots will be adopted to accomplish this trajectory tracking. This control scheme was derived from an improved Model Reference Adaptive Control (MRAC) method, and requires no knowledge of the robot dynamic model or parameter values for the robot, the payload, or the environment. As a result, the controller is robust to both model and parameter uncertainties, and is computationally fast
for on-line control applications with modest computing power.

The control algorithm computes the position control input $F_{p}$ as follows:

$$
F_{p}=d_{p}(t)+K_{p p}(t) E_{p}+K_{v p}(t) \dot{E}_{p}+C(t) x_{d}+B(t) \dot{x}_{d}+A(t) \ddot{x}_{d}
$$

where $E_{p}=x_{d}-x$ is the position tracking error, and $d_{p} \in R^{\prime}$ and $K_{p p}, K_{v p}, C, B, A \in R^{l \times l}$ are controller gains which are updated adaptively. The adaptation laws for these gains are provided in [22] and are not repeated here. Note that the control input $F_{p}$ is computed entirely based on the abserved performance of the manipulator. 
The control scheme computes the force control input $F_{f}$ using the following algorithm:

$$
F_{f}=d_{f}(t)+K_{I}(t) \int_{0}^{t} E_{f} d t+K_{p f}(t) E_{f}-K_{v f}(t) \dot{z}+P_{d}
$$

where $E_{f}=P_{d}-P$ is the force tracking error, and $d_{f} \in R^{j}$ and $K_{I}, K_{p f}, K_{v f} \in R^{j \times j}$ are controller gains which are updated adaptively. Again, the adaptation laws for these gains are provided in [22] and

control input $F_{f}$ is computed entirely based on the observed performance of the robot.

Finally, the position control input $F_{p}$ computed in (4) and the force control input $F_{f}$ computed in (5) are combined to form the Cartesian control input $F$ :

$$
F=\left[F_{p}^{T} \mid F_{f}^{T}\right]^{T}
$$

2.3 Redundancy Resolution Through Construction of $F \rightarrow T$ Map

Redundancy Resolution Through Construction of $\vec{F} \rightarrow T$ Map input must be mapped to an actuator torque vector $T$. The $F \rightarrow T$ mapping problem is underdetermined since $F \in R^{m}$ and $T \in R^{n}$ with $m<n$, so that it is at this stage of the control problem that the robot redundancy may be utilized to improve the robot's performance.

The problem of constructing an appropriate $F \rightarrow T$ map may be formulated in terms of inverting the known $T \rightarrow F$ map, which is unique even for redundant robots. The $T \rightarrow F$ map may be shown to be $[13,16]$

$$
F=\left(J H^{-1} J^{T}\right)^{-1} J H^{-1} T \equiv M(\theta) T
$$

where it is easily verified that $M \in R^{m \times n}$. Inversion of the $T \rightarrow F$ map (7) may be achieved in two ways:

1.) "direct" inversion of (7) using the theory of generalized inverses [23]

2.) "indirect" inversion of (7) by first augmenting both $M$ and $F$ with $r=n-m$ additional rows and then inverting the resulting fully determined system by standard methods

Each of these approaches is now briefly summarized. Additional details concerning each inversion method are provided in Sections 3 and 4 of this paper, and also in $[13,24]$.

The direct approach to inverting (7) has proven useful for realizing dynamic performance objectives, primarily because inverting (7) using generalized inverse theory readily permits optimization of objective functions involving joint torque $T$ and joint accelerations $\ddot{\theta}$. For example, the $F \rightarrow T$ map which minimizes the norm of the joint torque vector $\|T\|=\left(T^{T} T\right)^{1 / 2}$, subject to the constraint ( 7 ), may be easily derived using generalized inverses:

$$
T=H^{-1} J^{T}\left(J H^{-2} J^{T}\right)^{-1} J H^{-1} J^{T} F
$$

The indirect approach to inverting ( 7 ) has been utilized principally for realizing kinematic performance objectives. While the idea of augmenting $M$ and $F$ with $r$ additional rows to obtain a fully determined system is conceptually simple, selecting these additional rows in such a way that some desired performance objective is realized is more difficult. The process of augmenting $M$ in an appropriate manner can be simplified somewhat by choosing to augment $J$ instead. Let $J_{a}=\left[J^{T} \mid J_{c}^{T}\right]^{T} \in R^{n \times n}$ be the matrix that results from augmenting $J$ with $J_{c} \in R^{r \times n}$. Then, provided $J_{a}$ is nonsingular, replacing $J$ with $J_{a}$ in (7) allows this $T \rightarrow F$ map to be inverted by standard methods, yielding

$$
T=J^{T} F+J_{c}^{T} F_{c}
$$

where $F_{c} \in R^{r}$ is an appropriately chosen vector used to augment $F$. One method of specifying the terms $J_{c}$ and $F_{c}$ in (9) has been derived by Seraji [17], and is summarized in Section 4 of this paper.

\section{OPTIMIZATION OF THE DYNAMIC RESPONSE OF THE ROBOT}

One of the advantages of a redundant robot is the potential to use the "extra" DOF to improve the robot's dynamic response $[13,14,25]$. One approach to achieving this improved performance is to devise a strategy for allocating motion among the robot joints in such a way that the desired end-effector motion is tracked with minimum actuator torque. This strategy will increase the bandwidth of the robot for a given set of actuator torque limits, which in turn will lead to improved tracking of both position and force trajectories [26].

Local minimization of the (norm of the) joint torque vector required to provide the desired Cartesian control input $F$ is achieved in Section 2.3 using the direct approach to inverting the $T \rightarrow F$ map (7), and the result is given in (8). However, it has been found in previous investigations that local (in time) minimization of joint torques often leads to trajectories that are globally unstable $[9,27]$; thus implementation of the $F \rightarrow T$ map (8) may be undesirable.

An alternative approach to reducing joint torque requirements is to consider the following constrained optimization problem:

$$
\text { minimize } \int_{0}^{t_{g}} \frac{1}{2} \dot{\theta}^{T} H \dot{\theta} d t \quad \text { subject to the constraint } y=f(\theta)
$$


where $t_{f}$ is the trajectory completion time. Note that $(10)$ is a global optimization problem, and therefore its wolution should possess the desirable characteristics of a globally optimal solution, such as trajectory stability. Note also that minimizing total robot kinetic energy integrated over the entire trajectory, subject to the constraint of desired end-effector motion, should lead to a uniform reduction in joint torques and a corresponding uniform increase in dynamic response. The optimization problem (10) may be analyzed using the calculus of variations [28]. First, the intermediate function $L(\theta, \dot{\theta}, \lambda) \in R$ is constructed:

$$
L=\frac{1}{2} \dot{\theta}^{T} H \dot{\theta}+\lambda^{T}(t)[y-f(\theta)]
$$

where $\lambda \in R^{m}$ is the Lagrange multiplier vector. The necessary conditions on (11) for optimality of (10) are

$$
\frac{\partial L}{\partial \lambda}=0 \quad, \quad \frac{\partial L}{\partial \theta}-\frac{d}{d t} \frac{\partial L}{\partial \dot{\theta}}=0
$$

Substituting (11) into the necessary conditions (12) yields, after some simplification,

$$
\ddot{y}-J \ddot{\theta}-j \dot{\theta}=0 \quad, \quad H \ddot{\theta}+\dot{H} \dot{\theta}-J^{T} \lambda-\frac{1}{2} \partial\left(\dot{\theta}^{T} H \dot{\theta}\right) / \partial \theta=0
$$

The equations (13) may be solved for $\ddot{\theta}[13]$ :

$$
\ddot{\theta}=H^{-1} J^{T}\left(J H^{-1} J^{T}\right)^{-1}[\ddot{y}-j \dot{\theta}]-\left[I_{n}-H^{-1} J^{T}\left(J H^{-1} J^{T}\right)^{-1} J\right] H^{-1} V_{c c}
$$

where $I_{n} \in R^{n \times n}$ is the identity matrix. Note that the solution (14) to the problem (10) has been obtained, independently, by Kaxerounian and Wang [29].

Expressing the necessary condition for optimizing (10) as an $F \rightarrow T$ map may be achieved by substituting the joint-space dynamic model (3a) and the Cartesian-space dynamic model (3b) into the necessary condition (14), and simplifying the result:

$$
T=J^{T} F+\left[I_{n}-J^{T}\left(J H^{-1} J^{T}\right)^{-1} J H^{-1}\right]\left(V_{f}+G\right)
$$

A close approximation to the global minimum kinetic energy $F \rightarrow T$ map (15) may be obtained as follows. Observe that the operator $\left[I_{n}-J^{T}\left(J H^{-1} J^{T}\right)^{-1} J H^{-1}\right]$ projects the vector $V_{\ell}+G$ into the null-space of $J H^{-1}$ (this may be verified by pre-multiplying the projection $\left[I_{n}-J^{T}\left(J H^{-1} J^{T}\right)^{-1} J H^{-1}\right]\left(V_{f}+G\right)$ by $J H^{-1}$ and noting that the result is the zero vector). In fact, it is ahown in [24] that this operator projects the vector $V_{f}+G$ onto only a portion of the null-space of $J H^{-1}$, and that typically the resulting projection is small compared to the term $J^{T} F$. These results imply that the $F \rightarrow T$
map

$$
T=J^{T} F
$$

is a close approximation to the global minimum kinetic energy $F \rightarrow T$ map. Note that the map (16) is computationally efficient and requires no knowledge of the robot dynamic model.

In summary, it is hypothesized that utilizing the robot redundancy to construct the $F \rightarrow T$ map which minimizes robot kinetic energy integrated over the trajectory, subject to the constraint of desired end-effector motion, will lead to a uniform reduction in joint torques and a corresponding uniform increase in dynamic response. Moreover, the resulting robot trajectory should be stable because of the globally optimal nature of this $F \rightarrow T$ map. In view of the fact that the $F \rightarrow T$ map (16) is a close approximation to the minimum kinetic energy map (15), and possesses the desirable features of computational efficiency and robustness to dynamic model uncertainty, it is proposed that the map (16) be employed in the control algorithm. The performance of the control scheme (4)-(6) together with the $F \rightarrow T$ map (16) is examined through computer simulation in
Section 5.

\section{CONSIDERATION OF KINEMATIC PERFORMANCE OBJECTIVES}

In this Section, the control algorithm (4)-(6),(16) is modified so that the robot redundancy is used to simultaneously improve the robot's dynamic response and realize any specified kinematic performance objective.

It is shown in [18] that a redundant robot may be controlled to track a desired end-effector position/force trajectory and simultaneously satisfy an $r$-dimensional kinematic constraint of the form

$$
\psi(t)=g(\theta)
$$

where $g: R^{n} \rightarrow R^{r}$ and $\psi \in R^{r}$ defines the evolution of $g$. The control algorithm developed to achieve this desired performance computes the Cartesian control input $F$ using (4)-(6), and then maps this control input to the robot actuator torque $T$ as follows:

$$
T=J^{T} F+\rho(\partial g / \partial \theta)^{T} F_{c}
$$


where $\partial g / \partial \theta \in R^{r \times n}$ is the constraint Jacobian, $\rho \in R^{+}$was implicitly defined as $\rho=1$ in [18], and $F_{c} \in R^{r}$ is the constraint control input required to track the desired evolution of $(17)$, denoted as $\psi_{d}(t)$. The constraint control input $F_{c}$ is computed as

$$
F_{c}=d_{p}(t)+K_{p p}(t) E_{c}+K_{v p}(t) \dot{E}_{c}+C(t) \psi_{d}+B(t) \dot{\psi}_{d}+A(t) \ddot{\psi}_{d}
$$

where $E_{c}=\psi_{d}-\psi$ is the constraint tracking error, and the adaptive gains $d_{p} \in R^{r}$ and $K_{p p}, K_{u p}, C, B, A \in R^{r \times r}$ are updated based on the constraint tracking error $E_{c}$.

Observe that setting $\rho=0$ in (18) reduces that map to the (approximate) minimum kinetic energy $F \rightarrow T$ map (16), while setting $\rho=1$ in (18) causes the robot redundancy to be used to closely track the kinematic constraint (17). Thus the map (18) may be viewed as a modification of the map (16) to include the potential to satisfy kinematic constraints, and the parameter $\rho$ may be used to specify the relative importance of dynamic response and constraint tracking accuracy. In typical applications (e.g., obstacle avoidance, joint limit avoidance), the constraint (17) need not be tracked with the same accuracy as the end-effector task. Then $\rho$ can be chosen small, and adequate constraint tracking and improved dynamic response can be achieved simultaneously. The selection of an appropriate value for $\rho$ and the effect of this choice on the performance of the robot is quantified through example in Section 5.

The control algorithm (4)-(6), (17)-(19) provides a method for controlling a redundant robot so that end-effector position/force trajectory tracking and general kinematic constraint satisfaction are achieved simultaneously. This control scheme can be extended to include utilizing the redundancy to optimize general kinematic performance objectives. Let the general kinematic performance optimization problem be formulated as

$$
\text { maximize } G(\theta) \quad \text { subject to the constraint } y=f(\theta)
$$

where $G: R^{n} \rightarrow R$ may be constructed to represent a measure of any desired kinematic performance objective. The solution to $(20)$ can be obtained using Lagrange multipliers. Let the augmented scalar objective function $G^{*}(\theta, \lambda)$ be defined as

$$
G^{*}(\theta, \lambda)=G(\theta)+\lambda^{T}[y-f(\theta)]
$$

where $\lambda \in R^{m}$ is the vector of Lagrange multipliers. The necessary conditions for optimality of (20) may be written using (21):

$$
\begin{aligned}
& \partial G^{*} / \partial \lambda=0 \quad \Rightarrow \quad y=f(\theta) \\
& \partial G^{*} / \partial \theta=0 \quad \Rightarrow \quad \partial G / \partial \theta=J^{T} \lambda
\end{aligned}
$$

From (22), it is seen that a necessary condition for optimality of (20) is that $\partial G / \partial \theta \in R\left(J^{T}\right)$. This requirement may be written concisely as

$$
A \partial G / \partial \theta=0
$$

where $A \in R^{r \times n}$ is any matrix whose rows form a basis for the null-space of $J$. This result is a direct consequence of the fact what the row-space and the null-space of any matrix are orthogonal complements. Note that (23) can alternatively be obtained using gradient projection optimization theory [30], and that this approach was first proposed for redundancy resolution by Baillieul in his "extended Jacobian" method [4]. When $-G(\theta)$ is convex, the condition (23) is both necessary and sufficient to solve (20). This is of interest because in robotics applications it is usually possible to construct $G(\theta)$ so that $-G(\theta)$ is convex.

Observe that the optimality condition (23) is an $r$-dimensional kinematic equality constraint of the form (17) with $g=$ $A \partial G / \partial \theta$ and $\psi_{d}(t)=0$. Therefore the control law (4)-(6), (17)-(19) can be used for simultaneous end-effector trajectory tracking and optimization of any desired kinematic objective function $G(\theta)$. Indeed, assuming that $G(\theta)$ is defined (and differentiable), specification of the kinematic equality constraint that is to be tracked to achieve this optimization requires only that an appropriate $A$ matrix be constructed and that the calculations specified in (23) be performed. The matrix $A$ may be constructed in several ways; one formulation for $A$ is [13]

$$
A=\left[-J_{2}^{T}\left(J_{1}^{-1}\right)^{T} \quad \mid I_{r}\right]
$$

where $J_{1} \in R^{m \times m}$ and $J_{2} \in R^{m \times r}$ are the partitions of $J$ defined by $J=\left[\begin{array}{lll}J_{1} & \mid J_{2}\end{array}\right]$. The validity of the construction (24) for $A$ may be verified by observing that $A J^{T}=0$ and that the row rank of $A$ is $r$ for all $\theta$ due to the presence of the $I_{r}$ partition in (24).

Summarizing, the control algorithm (4)-(6), (17)-(19) can be extended to include utilizing the redundancy to solve the kinematic performance optimization problem (20) by setting $g=A \partial G / \partial \theta$ and $\psi_{d}=0$, where $A \in R^{r \times n}$ is given in (24). The comments made previously concerning the role of the parameter $\rho$ in the control law apply here as well, of course. The use of the control algorithm (4)-(6), (17)-(19) for the case in which the kinematic performance objective is the optimization of a kinematic objective function is illustrated in Section 5 . 


\section{SIMULATION RESULTS}

\subsection{Overview of Computer Simulation Study}

The Cartesian-space position/force control scheme for redundant robots given in (4)-(6), (17)-(19) is now applied to a direct-drive four-link planar robot in two computer simulation examples. The results presented here are samples selected from a comprehensive computer simulation study which was carried out to test the performance of the proposed controller. Note that the results given here are selected because they are typical of the larger study, and not because they represent the best performance obtainable with the proposed control law.

Consider the four-link robot in a horizontal plane shown in Figure 1. The robot parameters are link lengths $l_{1}=l_{2}=$ $l_{3}=l_{4}=1.0 \mathrm{~m}$, link masses $m_{1}=m_{2}=m_{3}=m_{4}=10.0 \mathrm{~kg}$, and joint viscous friction coefficients $c_{1}=c_{2}=c_{3}=$ $c_{4}=40.0 \mathrm{Nt} \cdot \mathrm{m} \cdot \mathrm{sec} ;$ the link inertias are modeled by thin uniform rods. The frictionless reaction surface is located parallel to the $x$-axis at $z=0.0$ and has a stiffness of $10^{4} \mathrm{Nt} / \mathrm{m}$. Note that in this example the base frame is chosen as the constraint frame, so that the position subspace and the force subspace are each of dimension one and correspond to the $x$ and $z$ axes, respectively. The robot dynamic model which relates joint torques $T \in R^{4}$ and joint angles $\theta \in R^{4}$ is given by

$$
T=H(\theta) \ddot{\theta}+V_{c c}(\theta, \dot{\theta})+V_{f}(\dot{\theta})+J_{f}^{T} P
$$

In the dynamic model (25) the numerical values for the inertia matrix $H \in R^{4 \times 4}$, Coriolis and centrifugal torque vector $V_{c c} \in R^{4}$ and viscous friction torque vector $V_{f} \in R^{4}$ may be found in [19]. Note that the gravity vector is orthogonal to the plane of motion of the robot, so that no gravity torques appear in (25). It must be emphasized that the dynamic model (25) is used only to simulate the robot behavior and is not used in the control law formulation.

In the aimulation study, the performance of the control scheme (4)-(6), (17)-(19) is evaluated through comparison with a commonly proposed approach to redundancy resolution, the inertia-weighted pseudoinverse approach [9]. Specifically, the performance of the proposed controller is compared with the performance of a controller which resolves the robot redundancy
as follows:

$$
\ddot{\theta}=H^{-1} J^{T}\left(J H^{-1} J^{T}\right)^{-1}[\ddot{y}-j \dot{\theta}]
$$

To allow a meaningful comparison to be made between the control law (4)-(6), (17)-(19) and the redundancy resolution scheme (26), the inveree kinematics algorithm (26) must be implemented as an equivalent $F \rightarrow T$ map. This equivalent $F \rightarrow T$ map may be derived using the same approach taken when rewriting the inverse kinematics algorithm (14) as the equivalent $F \rightarrow T$
map (15), and yields the following result:

$$
T=J^{T} F+\left[I_{n}-J^{T}\left(J H^{-1} J^{T}\right)^{-1} J H^{-1}\right]\left(V_{c c}+V_{J}\right)
$$

The $F \rightarrow T$ map (27) may be combined with the control scheme (4)-(6) to yield a pseudoinverse-based position/force controller this controller resolves the robot redundancy exactly as prescribed in the inverse kinematics algorithm (26). Note that in deriving the $F \rightarrow T$ map (27) it is implicitly assumed that $G=0$, since this is the case in the simulation study.

We now turn to the discussion of two computer simulation examples. Throughout this discussion, the control law (4)-(6), (17)-(19) will be referred to as the proposed controller while the control scheme given by (4)-(6) together with the $F \rightarrow T$ map (27) will be called the weighted pseudoinverse (WP) controller. Additionally, in these simulations, the unit of length is meter, the unit of angle is radian, the unit of force is Newton, and the unit of time is second.

\subsection{Simulation 1}

The task requirements for this simulation are to have the robot end-effector track a straight-line position/constant force trajectory while utilizing the redundancy to improve the dynamic response of the robot. The desired end-effector position trajectory is $x_{d}(t)=2.0+A_{o}-A_{o} \cos \omega t$, for $t \subset[0, \pi / \omega]$ and for different values of the trajectory parameters $A_{o}$ and $\omega$. The desired end-effector /environment contact force is $P_{d}(t)=10.0$, for $t \subset[0, \pi / \omega]$. The initial configuration of the robot is $\theta(0)=\left[\begin{array}{llll}\pi / 3 & -2 \pi / 3 \quad 2 \pi / 3 & -2 \pi / 3\end{array}\right]^{T}$, and the robot is initially at rest.

The proposed controller and the WP controller each accomplishes the required position/force trajectory tracking by employing the Cartesian control algorithm (4)-(6). The desired position trajectory is tracked using a scalar version of the position control algorithm (4), and the desired force trajectory is tracked using a scalar version of the force controller (5). The position control input $F_{p}$ and force control input $F_{f}$ are combined to form $F$ as prescribed in (6).

Redundancy resolution is achieved when mapping the Cartesian control input $F$ (computed in (4)-(6)) to joint actuator torque $T$. The map used in the proposed controller for increasing the robot's dynamic response in a stable manner is given in (18) with $\rho=0$. The $F \rightarrow T$ map used by the WP controller is given in (27). The algorithm (4)-(6) together with the appropriate $F \rightarrow T$ map is applied to the dynamic model (25) through computer simulation on a SUN $3 / 50$ computer with a
sampling period of one millisecond.

In the first simulation, the end-effector trajectory parameters are assigned the values $A_{o}=0.5$ and $\omega=0.25$. The results of the simulation are shown in Figures 2a-2c, and indicate that both controllers perform well. This is as expected, because the required end-effector motion is slow and of moderate length.

In the next simulation, the end-effector trajectory is made both longer and faster by choosing trajectory parameter values of $A_{o}=0.8$ and $\omega=1.25$. The results of the simulation are given in Figures $2 \mathrm{~d}$ and $2 \mathrm{e}$, and show that the WP controller requires much larger torques than the proposed controller, and yet achieves poorer tracking accuracy. 


\subsection{Simulation 2}

This simulation illustrates the proposed controller's capability to use the robot redundancy to improve dynamic response and achieve a desired kinematic performance objective simultaneously. The desired end-effector position/force trajectory to be tracked in this simulation is quantified by $x_{d}(t)=\sqrt{3}+0.8-0.8 \cos 1.25 t$ and $P_{d}(t)=10.0$, for $t \subset[0,4 \pi / 5]$. The kinematic performance objective to be achieved simultaneously with improved dynamic response is the maximization of the "manipulability measure" $w: R^{n} \rightarrow R^{+}$, defined by Yoshikawa as follows [1]:

$$
w(\theta)=\left(\operatorname{det}\left[J J^{T}\right]\right)^{1 / 2}
$$

Briefly, it has been proposed by Yoshikawa [1] and others that utilizing robot redundancy to maximize manipulability may be an effective means of increasing robot dexterity and avoiding kinematic singularities.

The proposed controller and the WP controller each accomplishes the required position/force trajectory tracking by employing the Cartesian control algorithm (4)-(6), as described in Section 5.2 for Simulation 1. Redundancy resolution is achieved in these controllers when mapping the Cartesian control input $F$ to joint torque $T$. The map used in the proposed controller for increasing dynamic response and manipulability simultaneously is given in (17)-(19) with $g=A \partial w / \partial \theta$ and $\psi_{d}(t)=0$, where the matrix $A$ is constructed as in (24). The parameter $\rho$, which specifies the relative importance of increasing dynamic inceasing manipulability, is chosen (heuristically) as $\rho=0.1$. A measure of how effectively this proposed controller increases dynamic response is obtained through comparison with the WP controller, which maps control input $F$ computed in (4)-(6) to joint torque $T$ using (27). The effectiveness of the proposed controller at increasing manipulability is evaluated by comparing the evolution of $w(\theta)$ over the trajectory to the maximum possible values for $w$ given the end-effector trajectory specified in this simulation.

In the simulation, the algorithm (4)-(6) together with the appropriate $F \rightarrow T$ map is applied to the robot dynamic model (25) through computer simulation on a SUN $3 / 50$ computer with a sampling period of one millisecond. It can be shown that in order to maximize manipulability by tracking the optimality condition $A \partial w / \partial \theta=0$, it is necessary that the initial robot configuration be the maximum manipulability configuration corresponding to the initial end-effector position [24]. One method of obtaining the optimal initial configuration $\theta^{*}(0)$ is to integrate the differential equation

$$
\dot{\theta}=\left[I-J^{T}\left(J J^{T}\right)^{-1} J\right] \partial w / \partial \theta
$$

until it reaches equilibrium. The starting point for the integration may be any configuration $\theta$ which places the end-effector in the desired initial position, and the equilibrium configuration of (29) is the optimal configuration $\theta^{*}$. Using this algorithm, the optimal initial configuration of the robot is obtained as $\theta(0)=\left[\begin{array}{llll}1.697202 & -1.570791 & -0.252815 & -1.570791\end{array}\right]^{T}$. In this simulation the robot is initially at rest.

The results of the simulation are shown in Figures 3a-3d. These results indicate that the WP controller requires much arger torques than the proposed controller, and exhibits poorer tracking accuracy. Additionally, the results show that the manipulability is very nearly maximum over the entire trajectory. Thus the proposed controller accurately tracks the required trajectory and successfully increases both the robot's dynamic response and manipulability measure over the entire trajectory.

\section{CONCLUSIONS}

This paper presents a Cartesian-space position/force control scheme for redundant robots. The proposed control strategy is to partition the control problem into a nonredundant position/force trajectory tracking problem and a redundant mapping problem between Cartesian control input $F$ and robot actuator torque $T$. The underdetermined nature of the $F \rightarrow T$ map is exploited to allow the redundancy to be effectively utilized directly in Cartesian-space. Computer simulation results are given for a four DOF planar redundant robot under the control of the proposed algorithm, and demonstrate that accurate position/force trajectory tracking and effective utilization of redundancy can be achieved simultaneously with the controller.

\section{ACKNOWIEDGEMENTS}

The research described in this paper was supported by Sandia National Laboratories under contracts 06-5630 and 05-9859. The authors gratefully acknowledge the many helpful conversations on topics of this paper with Homayoun Seraji of the Jet Propulsion Laboratory and Patrick Eicker of Sandia National Laboratories.

8. REFERENCES

1. Yoshikawa, T., “Analysis and Control of Robot Manipulators with Redundancy", Robotics Research: The First Intern. Symp., eds. Brady and Paul, Cambridge, MA., MIT Press, 1984, pp. 735-748

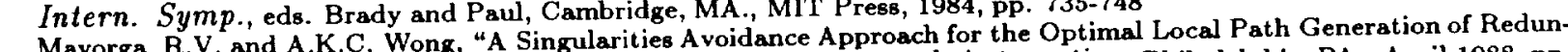
dant Manipulators", Proc. IEEE Intern. Conf. on Robotics and Automation, Philadelphia, PA., April 1988, pp.

3. Maciejewski, A.A. and C.A. Klein, "Obstacle A voidance for Kinematically Redundant Manipulators in Dynamically Varying Environments", Intern. J. Robotics Research, Vol. 4, No. 3, 1985, pp. 109-117

4. Baillieul, J., "Avoiding Obstacles and Resolving Kinematic Redundancy", Proc. IEEE Intern. Conf. on Robotics and Automation, San Francisco, CA., April 1986, pp. 1698-1704

5. Liegeois, A., "Automatic Supervisory Control of the Configuration and Behavior of Multibody Mechanisms", IEEE Trans. Sys., Man, and Cyber., Vol. SMC-7, No. 12, 1977, pp. 868-871

6. Klein, C.A. and C.H. Huang, "Review of Pseudoinverse Control for Use with Kinematically Redundant Manipulators", IEEE Trans. Sys., Man, and Cyber., Vol. SMC-13, No. 3, 1983, pp. 245-250 
7. Baillieul, J., J.M. Hollerbach and R. Brockett, "Programming and Control of Kinematically Redundant Manipulators" Proc. 29rd Conf. on Decision and Control, Las Vegas, NV., December 1984, pp. 768-774

8. Chiu, S.L., "Kinematic Characterization of Manipulators: An Approach to Defining Optimality", Proc. IEEE Intern. Conf. on Robotics and Automation, Philadelphia, PA., April 1988, pp. 828-833

9. Hollerbach, J.M. and K.C. Suh, "Redundancy Resolution of Manipulators Through Torque Optimization", IEEE J. of Robotics and Automation, Vol. RA-3, No. 4, 1987, pp. 308-316

10. Colbaugh, R. and K. Glass, "Real Time Resolution of Robot Manipulator Redundancy Through Joint Torque Optimization", Proc. ISMM Intern. Symp. on Computer Applications, Honolulu, HA., February 1988, pp. 168-172

11. Vukobratovic, M. and M. Kircanski, "A Dynamic Approach to Nominal Trajectory Synthesis for Redundant Manipulators", IEEE Trans. Sys., Man, and Cyber., Vol. SMC-14, No. 4, 1984, pp. 1016-1021

12. Colbaugh, R. and K. L. Phillips, "Energy Oplimal Trajectory Planning for Robots", Space Nuclear Power Systems
1988, Orbit Book Company, 1988

13. Colbaugh, R., "Dynamic Performance Optimization of Redundant Robot Manipulators", Preprints 842nd Meeting of the American Mathematical Society, Las Cruces, NM., April 1988 14. Egeland, O., "Task-Space Tracking with Redundant Manipulators", IEEE J. of Robotics and Automation, Vol. RA-3,
No. 5, 1987, pp. 471-475

15. Hsu, P., J. Hauser and S. Sastry, "Dynamic Control of Redundant Manipulators", Proc. IEEE Intern. Conf. on Robotics and Automation, Philadelphia, PA., April 1988, pp. 183-187

16. Khatib, O., "A Unified Approach for Motion and Force Control of Robot Manipulators: The Operational Space Formulation", IEEE J. of Robotics and Automation, Vol. RA-3, No. 1, 1987, pp. 43-53

17. Seraji, H., "Gonfiguration Control of Redundant Manipulators", Proc. NATO Advanced Research Workshop on Robots with Redundancy, June 1988

18. Seraji, H., R. Colbaugh, K. Glass and T. Lee, "Experimental and Simulation Studies of Configuration Control for Redundant Robots", submitted to 1989 IEEE Intern. Conf. on Robotics and Automation, Scot tsdale, AZ., May 1989

19. Colbaugh, R., H. Seraji and K. Glass, "Obstacle A voidance for Redundant Robots Using Configuration Control" (to appear)

20. Raibert, M. H. and J. J. Craig, "Hybrid Position/Force Control of Manipulators", ASME J. Dyn. Sys., Measurement, and Control, Vol. 102, No. 2, 1981, pp. 126-133

21. Tarn, T.J., A.K. Bejczy and X. Yun, "Robot Arm Force Control through System Linearization by Nonlinear Feedback", Proc. IEEE Intern. Conf. on Robotics and Automation, Philadelphia, PA., April 1988, pp. 1618-1625

22. Seraji, H., “Adaptive Force and Position Control of Manipulators", Journal of Robotic Systems, Vol. 4, No. 4, 1987,
pp. 551-578

23. Lawson, C. L. and R. J. Hanson, Solving Least Squares Problems, Prentice-Hall, Englewood Cliffs, NJ., 1974

24. Colbaugh, R. and K. Glass, "Cartesian Position/Force Control of Redundant Robots", Final Report for Contract 06-5690 with Sandia National Laboratories, Engineering Research Center, New Mexico State University, November

25. Salisbury, J.K. and J.D. Abramowitz, "Design and Control of a Redundant Mechanism for Small Motion", Proc. IEEE Intern. Conf. on Robotics and Automation, St. Louis, MO., March 1985, pp. 323-328 26. Whitney, D.E., "Historical Perspective and State of the Art in Robot Force Control", Intern. J. of Robotics Research,
Vol. 6, No. 1, Spring 1987, pp. 3-14

27. Kazerounian, K. and A. Nedungadi, "An Alternative Method for Minimization of Driving Forces in Redundant Manipulators", Proc. IEEE Intern. Conf. on Robotics and Automation, Raleigh, NC., April 1987, pp. 1701-1706

28. Petrov, I., Variational Methods in Optimum Control Theory, Academic Press, New York, 1968

Intern. J. Robotics Research, 1988, Vol. 7, No. 5, pp.3-12

30. Kirk, D.E., Optimal Control Theory, Prentice-Hall, Englewood Clifis, NJ., 1970

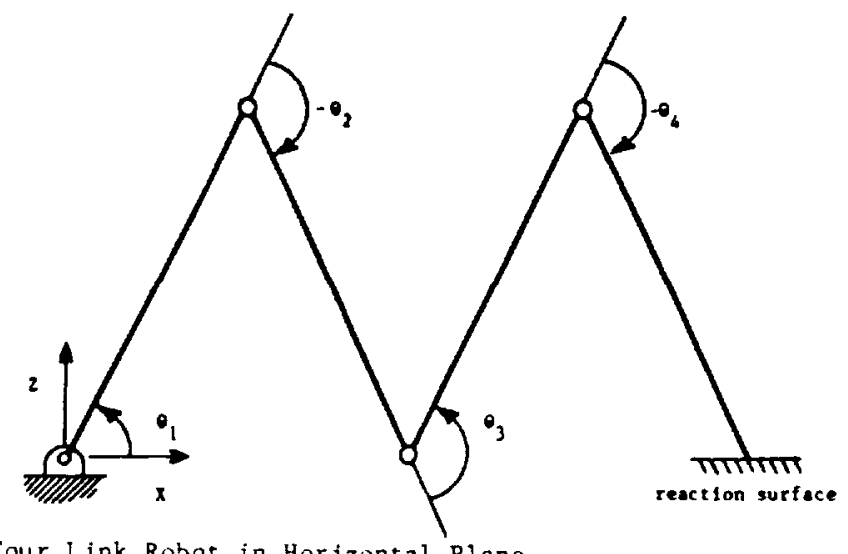

Figure 1. Four Link Robat in Horizontal plane 


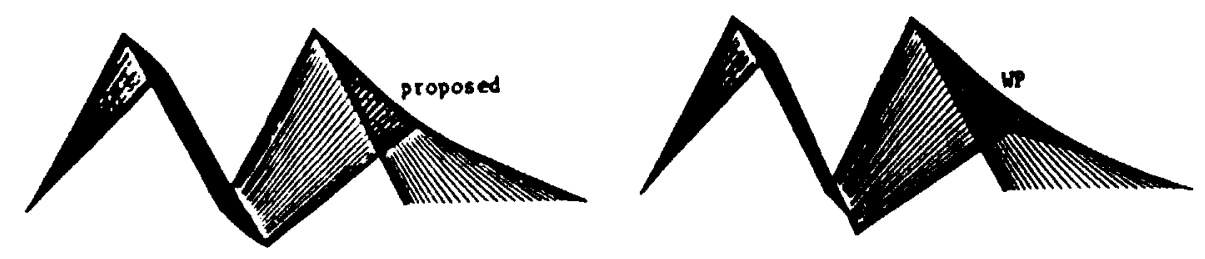

Figure 2 . Robot Configurations in Simulation $1\left(\Lambda_{0}=0.5,0=0.25\right)$

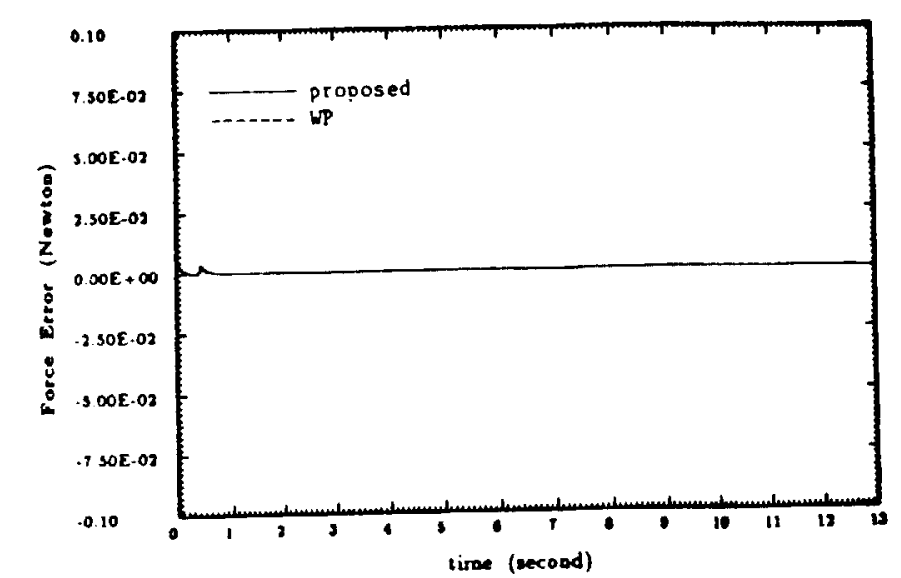

Figute 2b. End-Effector/Environment Contact Force Error in Simulation 1 $\left(\lambda_{0}=0.5, * 0.25\right)$

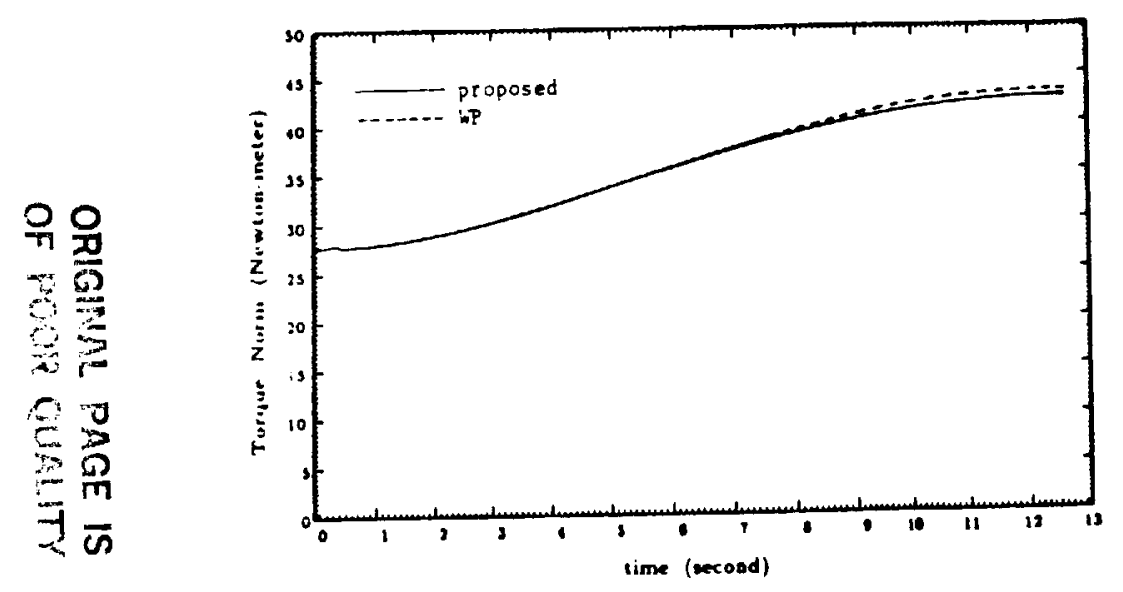

Figureic. Vorn of joint forque vector in simulation $1\left(\Lambda_{0}=0.5, w=0.25\right)$

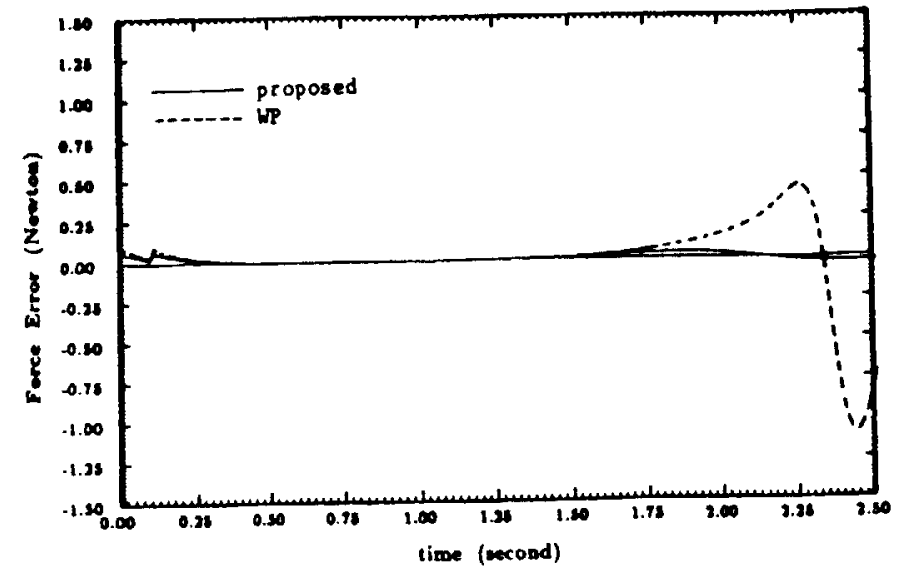

Figure 2d. End-Effector/Environment Contact Force Error in Simulation $\left(A_{0}=0.8, v=1.25\right)$

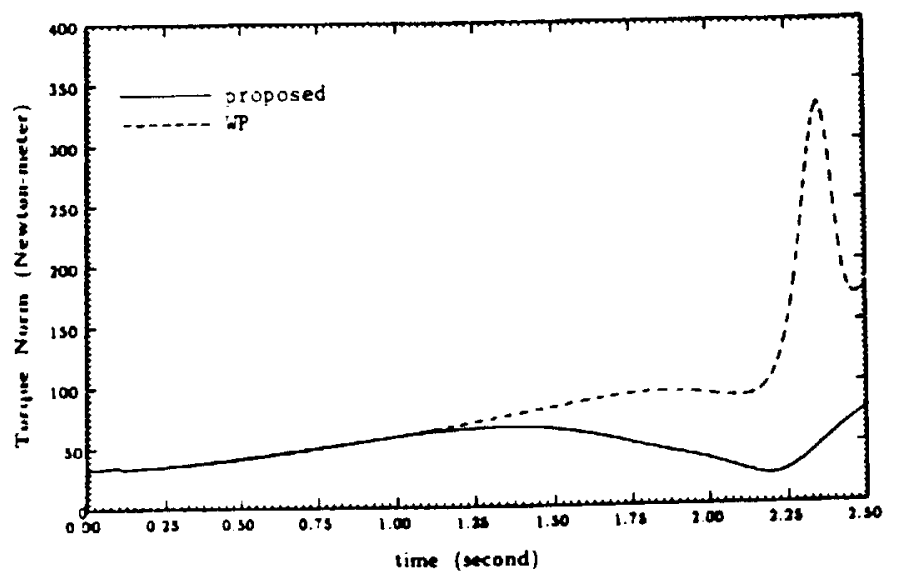

Figure 2e. Norm of Joint torque vector in Simulation $1\left(A_{0}=0.8, *=1.25\right)$ 


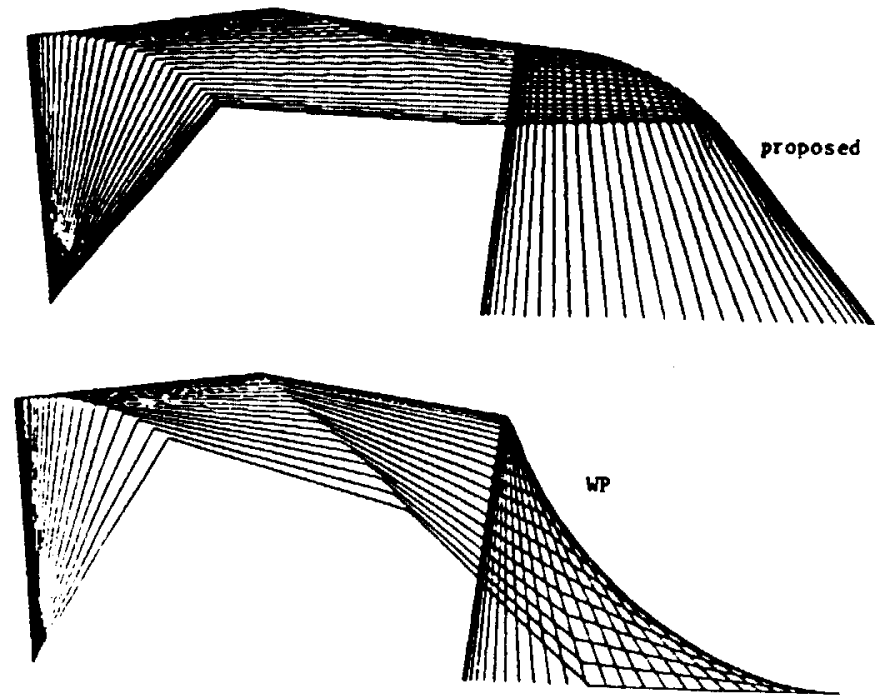

บั

Figure 3a. Robot configurations in Sioulation 2

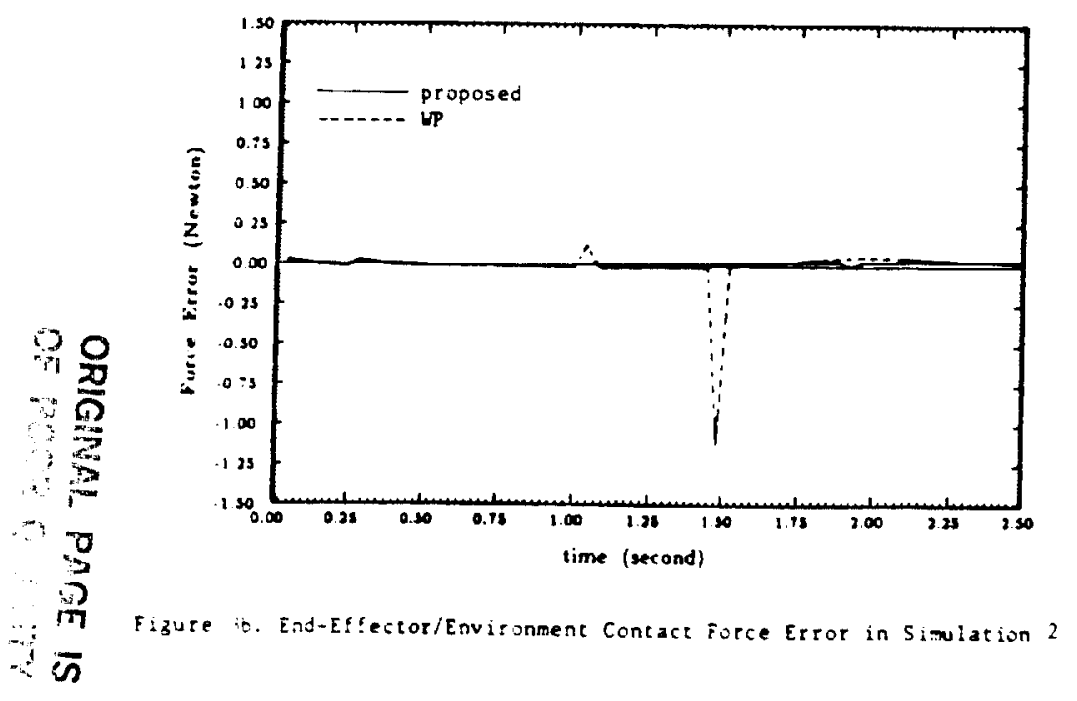

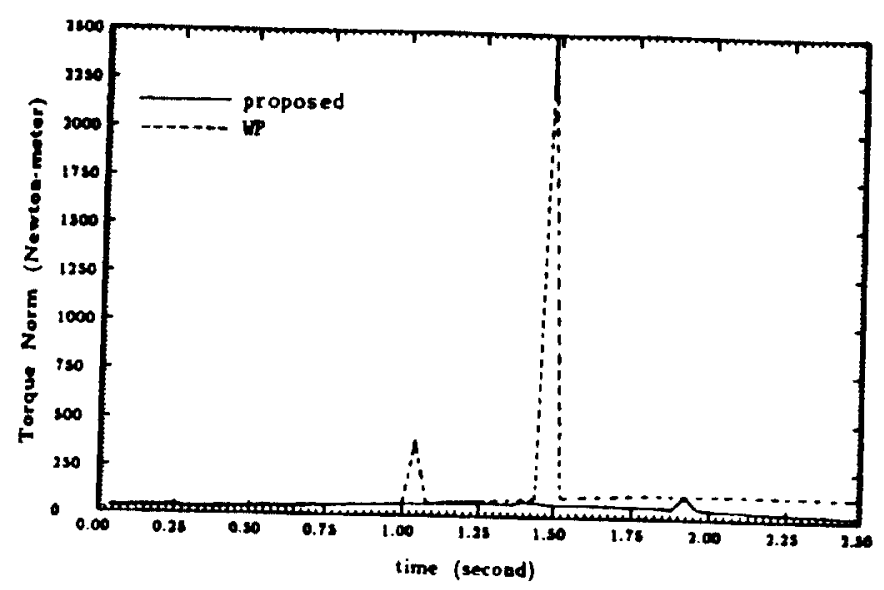

Figure 3c. Norm of Joint Torque Vector in Simulation 2

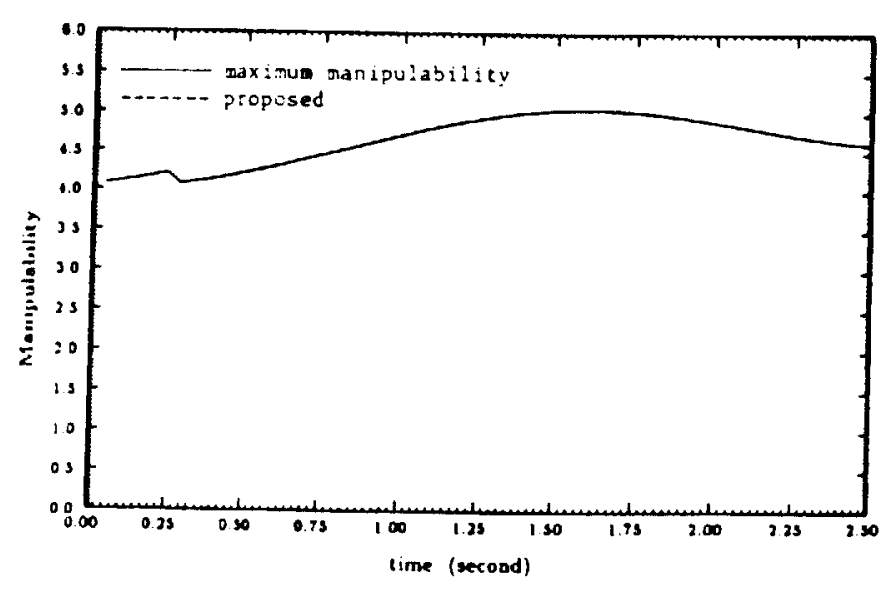

Figure d. Variation of Manipulability in Simulation? 